\title{
Response to comments on 'Methylphenidate improves response inhibition but not reflection impulsivity in children with attention deficit hyperactivity disorder (ADHD)'
}

\author{
Elise E. DeVito • Barbara J. Sahakian
}

Received: 13 October 2008 / Accepted: 15 October 2008 / Published online: 5 November 2008

(C) Springer-Verlag 2008

We thank Dr. Ghanizadeh for his interest in our study.

In his commentary, Dr. Ghanizadeh pointed out that psycho-stimulants may detrimentally effect brain development, and as such, the study should be repeated in drug-naïve children with ADHD. It is certainly true that the effects of MPH may be different in children who are drug-naïve versus those who have been previously stabilised on MPH. However, our study aims were to determine how the drug affected aspects of impulsivity and decision-making in children with ADHD who benefit clinically from MPH. We found that medication improved their response inhibition but did not improve their quality of decision-making (as operationalised by the Information Sampling Task). Furthermore, Dr. Ghanizadeh's concern regarding possible abnormal brain development is not a plausible mechanism to explain the MPH effects, since we used a within-subject design for those analyses.
Dr. Ghanizadeh also wrote that "intermediate to longterm psycho-stimulant medication may cause anxiety (Vance et al. 1999)" and suggested that this may have accounted in part for the performance of the children with ADHD versus healthy controls. To assess whether the healthy control and ADHD children felt different baseline levels of anxiety, we compared their visual analogue scale ratings of 'calmness', which is a composite score of how they rated themselves on subscales of "tense" versus "relaxed", "calm" versus "excited" and "shy" versus "outgoing". The ADHD group on their placebo visit did not significantly differ from the healthy controls on selfrated 'calmness' prior to testing $(F=1.10, p=0.302)$ or at the end of the testing session $(F=0.14, p=0.713)$. So, subjective levels of anxiety do not appear to explain the group differences.

E. E. DeVito $(\bowtie) \cdot$ B. J. Sahakian

Department of Psychiatry, University of Cambridge,

Box 189, Addenbrooke's Hospital,

Cambridge CB2 2QQ, UK

e-mail: eed24@medschl.cam.ac.uk 